Article

\title{
An Egocentric Network Contact Tracing Experiment
}

\author{
Andrew Pilny ${ }^{1 *}$ and C. Joseph Huber ${ }^{2}$ \\ 1 University of Kentucky; andy.pilny@uky.edu \\ 2 University of Kentucky; Joseph.Huber@uky.edu \\ * Correspondence: andy.pilny@uky.edu; Tel.: +1-859-257-8676 (F.L.)
}

\begin{abstract}
Contact tracing is one of the oldest social network health interventions used to reduce the diffusion of various infectious diseases. However, some infectious diseases like COVID-19 amass at such a great scope that traditional methods of conducting contact tracing (e.g., face-to-face interviews) remain difficult to implement, pointing the need to develop reliable and valid survey approaches. The purpose of this research is to test the effectiveness of three different egocentric survey methods for extracting contact tracing data: (1) a baseline approach, (2) a retrieval cue approach, and (3) a context-based approach. A sample of 397 college students were randomized into one of each condition and were prompted to anonymously provide contacts and populated places visited from the past four days. After controlling for various demographic, social identity, psychological, and physiological variables, participants in the context-based condition were significantly more likely recall more contacts (medium effect size) and places (large effect size) than the other two conditions. Theoretically, the research supports suggestions by field theory that assume network recall can be significantly improved by activating relevant activity foci. Practically, the research contributes to developing innovative social network data collection methods for contract tracing survey instruments.
\end{abstract}

Keywords: contact tracing; ego networks; experimental design; social networks; field theory

\section{Introduction}

Because infectious diseases like COVID-19 can be spread through respiratory droplets from being near and communicating with infected individuals, researchers have taken advantage of contact tracing to help mitigate the spread of the virus. Contact tracing is the process of retrospectively identifying persons who may have made interpersonal contact with a confirmed infectious individual [1]. The overall logic of contact tracing is to prevent individuals who made contact with the focal individual from further spreading the disease themselves and thus, breaking chains of infection within the larger system.

At its core, contact tracing is a form of egocentric network analysis (ENA) and thus, one type of social network intervention to influence public health. ENA is the study of individuals (i.e., egos) and people in their immediate social environment (i.e., alters). Conducting a reliable and valid ENA study is challenging, and there is a rich academic literature dedicated to improving the rigor behind these efforts [2,3]. Indeed, the effectiveness of contact tracing is only as good as the data that can be extracted. If ideal standards are not followed for collecting contact tracing data, then most efforts to take advantage of this data will likely fall short. Potential at-risk contacts may never be discovered for treatment and they could continue to propagate COVID-19. Likewise, hotspots and clusters are likely not to be discovered, preventing the public from being informed to follow social distancing. Moreover, recent simulation research has shown that in order for contact tracing to be effective for COVID-19, individuals must be able to successfully recall at least $80 \%$ of their contacts [4]. As such, the purpose of the proposed research is to test the effectiveness of three different egocentric survey methods for extracting contact tracing data. 


\subsection{A Survey-Based Approach for Contact Tracing}

In traditional egocentric network research, face-to-face interviews are often considered the goldstandard data collection mode [3]. The reasoning primarily has to do with reduced cognitive burden for the participant. For instance, telephone interviews or video conferencing introduce auditory and visual challenges (e.g., noise quality, background activities) and survey approaches introduce challenge with participants having to correctly navigate an egocentric design without assistance, which is more complex than traditional Likert-style questions [2] each of which are cognitive burdens notably absent in face-to-face interviewing.

However, there are growing challenges to implementing face-to-face interviews for contact tracing with respect to infectious diseases like COVID-19. First, face-to-face interviews are risky endeavors because the interviewer may put themselves at risk for contracting the infectious disease, especially when diagnoses specifically require a patient to quarantine. Second, interviewers need to be appropriately trained and inevitably vary on how skilled they are, resulting in interviewer effects [3]. As such, it is not surprising that there is a shortage of contact tracers appropriately trained to do such research [5]. Finally, as technology mediums continue to alter social environments [6], preferences for communication modalities must be continually monitored. For instance, in the food service industry, more people are using mobile food delivery apps on smartphones rather than calling in orders directly to restaurants [7].

These challenges to collecting contact tracing data via face-to-face interviews points towards the need to developing a reliable survey approach that could be instantly administered and standardized across various contexts. Following past egocentric network research, we examine the impact of three different survey designs: (1) baseline, (2) retrieval cue, and (3) context-based approaches. Each is described below.

\subsection{Theory and Hypothesis Development}

Perhaps the most important obstacle for egocentric data collection is recall and forgetting [3]. For the ego, contract tracing can be a cognitively intensive task. Especially when an individual has just learned that they are infected with a potentially fatal virus, it can be easy to forget nonchalant interactions from past several days. Furthermore, data from egocentric designs rely on self-reported network interactions, which are cognitive representations that may differ from actually observed interactions (for a review, see [8]). Moreover, prior literature has suggested that successful recall (i.e., remembering all of your contacts) is influenced by a number of factors because contacts tend be cognitively stored in clusters or what Brashears and Quintane [9] call chunked substructures. Likewise, there may be individual differences in recall pertaining to demographic and social identity factors like gender, age, race [10, 11] and psychological factors like mood and well-being [12, 13].

A baseline approach to surveying individuals about their contacts would simply just ask them about who they made contact with in a given period of time. This is commonly known as the interaction approach [13] because the goal is to find simple interactions rather than socially constructed relationships like friendship or individuals who provide important resources for the ego. For instance, Mossong and colleagues [15] define a contact as "EITHER a two-way conversation with three or more words in the physical presence of another person, OR physical skin-to-skin contact (for example a handshake, hug, kiss or contact sports)." Then, they ask each participant to "Write down every person that you contact during the day, regardless of whether the contact was long or short, and whether you know the person or not." In addition to baseline approaches, other more complex methods for ENA exist as well.

The retrieval cue approach for ENA was formalized by Hsieh [10]. This approach assumes that the "successful recall of an event depends primarily on how well the retrieval cues match the event's representations in one's memory organization" (p. 3). A retrieval cue is any additional piece of information that helps an ego remember a past event. The retrieval cue approach mirrors Tulving's [16] theory of cue-dependent forgetting, which assumes that forgetting, the inability to recall something in the present that could be recalled in the past, does not mean that the memory is lost, but only 
temporarily inaccessible. In Hsieh's study, participants were randomly assigned to a retrieval cue condition in which they were instructed to look at their (1) cellphone contact list, (2) last 30 emails, and (3) friend list on Facebook and Twitter as retrieval cues. Hsieh's results found that this approach yielded more contacts than a baseline approach.

Beyond contacts, it is also important for egos to remember populated places because individuals could have made contact with strangers whom they may not remember (e.g., a restaurant server). Likewise, overlapping data from multiple infected individuals who visited the same location could reveal 'hotspots' that may be useful for public health purposes. As such, we hypothesize:

Hypothesis 1. A retrieval cue approach will elicit more (a) contacts and (b) places visited than a baseline approach.

The context-based name generator was formalized by Bidart and Charbonneau [17]. The contextbased name generator begins by asking individuals about the social context cues of everyday life (e.g., work activity, shopping, home life). Then, once relevant contexts are triggered, contact names corresponding to those contexts are generated by the individual (see also [18]). As Bidart and Charbonneau explain, the context-based approach is motivated by field theory [19]. Field theory regards 'situated action' as a key unit of analysis (i.e., action that is not stripped away from a larger context). One of the premises of field theory is that most interpersonal interaction occurs because of shared activity foci. Activity foci are simply "aspects of extra-network social structure that systematically produce patterns in networks" and include "social, psychological, legal, or physical entities around which activities are organized" [19] (p. 1016). Indeed, subsequent research has shown that priming individuals with social context can help improve network recall [20,11]. As such, we hypothesize:

Hypothesis 2. A context-based approach will elicit more (a) contacts and (b) places visited than a baseline approach.

Finally, it is important to ask about whether or not a retrieval cue or context-based approach will significantly yield more contacts and places. To our knowledge, no study has specifically compared these two approaches and there exists little theoretical basis for expecting more or fewer differences. Thus, we keep this comparison as a research question:

Research Question 1. How do retrieval cue or context-based approaches differ in their ability to elicit more (a) contacts and (b) places visited?

\section{Materials and Methods}

\subsection{Design}

The experiment was a one-way between-subjects design. The related manipulation was contact elicitation, with three levels (baseline approach, retrieval cue approach, and context-based approach). Other unrelated control variables were uniformly collected across all participants (see Measures). There were two primary dependent variables: (1) unique contacts and (2) placed visited. An a priori power analysis suggests that at least 159 participants are needed to detect medium-sized effects.

\subsection{Participants}

To recruit participants for the experiment, we used a College wide research participation pool from a large southern University. A University sample was chosen in order to control for geographic policy differences (e.g., different counties having varying levels of stay-at-home policies) and the increasing relevance of college students' role in spreading COVID-19 [21]. The inclusion criteria required that students must be enrolled in participating classes where they are required to participate in at least two studies during that semester, which counts for $5 \%$ of their total grade. Alternative assignments are available if the students decline to consent in any of the available studies in the 
research pool. The University currently studied still had the majority of its classes face-to-face and the county had minimal restrictions regarding business openings (e.g., restaurants capped at 50\%, bars to close at midnight, masks required).

The study launched on September 18, 2020 and concluded on November 16, 2020. The reason for terminating the study on November 16, 2020 is because the state and county were soon to enact new COVID-19 restrictions, which may affect rates of interpersonal contact and places visited. After removing 18 cases of missing data (i.e., more than half of items were missing), the final sample size was 397: 138 in the baseline condition, 127 in the retrieval cue condition, and 132 in the context-based condition. The participants aged ranged from 18 to 42 , with an average age of $19.94(S D=2.35)$. Regarding gender, 262 (65.8\%) identified as female, $135(33.9 \%)$ as male, and one $(0.03 \%)$ preferred not to disclose. Finally, 305 (76.6\%) identified white, 50 (12.6\%) as African American, $17(4.30 \%)$ as Hispanic, $10(2.50 \%)$ as Asian, $1(0.03 \%)$ as Native Hawaiian or other Pacific Islander, and $15(3.80 \%)$ as Other (see Table 1).

Table 1. Correlations and descriptive statistics

\begin{tabular}{lllllllllll}
\hline & $\mathbf{( 1 )}$ & $\mathbf{( 2 )}$ & $\mathbf{( 3 )}$ & $\mathbf{( 4 )}$ & $\mathbf{( 5 )}$ & $\mathbf{( 6 )}$ & $\mathbf{( 7 )}$ & $\mathbf{( 8 )}$ & $\mathbf{( 9 )}$ & $\mathbf{( 1 0 )}$ \\
\hline Contacts (1) & & & & & & & & & & \\
Places (2) & .42 & & & & & & & & & \\
Gender (3) & .07 & .18 & & & & & & & & \\
Age (4) & .04 & .01 & -.05 & & & & & & & \\
Mood (5) & .09 & .07 & -.04 & .01 & & & & & & \\
Well-being (6) & .09 & .08 & -.04 & -.04 & .62 & & & & & \\
Physical functioning (7) & -.06 & -.10 & .14 & -.05 & -.06 & -.10 & & & & \\
Mask efficacy (8) & -.11 & -.09 & .06 & .03 & -.04 & -.09 & -.01 & & & \\
COVID-worry (9) & -.15 & -.12 & .06 & -.01 & -.12 & -.17 & .09 & .39 & & \\
COVID-serious (10) & -.13 & -.08 & .20 & .05 & -.05 & -.12 & .02 & .52 & .49 & \\
\hline Mean & 10.98 & 6.55 & 1.66 & 19.94 & 3.83 & 3.84 & 1.05 & 4.26 & 2.22 & 7.17 \\
Median & 9.00 & 6.00 & 2.00 & 20.00 & 3.88 & 3.88 & 1.00 & 4.00 & 2.00 & 7.00 \\
SD & 8.16 & 3.41 & .47 & 2.35 & .63 & .64 & .19 & .85 & .98 & 2.24 \\
\hline
\end{tabular}

\subsection{Apparatus}

The experiment was conducted online using Qualtrics. Participants were required to complete the study over a computer and laptop in the setting of their choice (i.e., at home). Students were not able to participate using a mobile device. Our decision to disallow mobile devices stemmed from the required textual input needed for providing contacts and places visited, which may be more difficult to supply over a mobile device.

\subsection{Procedure}

Participants took part in the experiment individually over a computer at the setting of their choice. When a student logs in to the research participation pool website, there are a variety of studies they can choose to complete. The research pool website includes the name of the study, which read "Mock Contact Tracing Study". A brief description of the study below the title included the text "This study will ask if you can remember, using anonymous pseudonyms, the people and places you have visited from the last four days".

If a student clicked to participate in the study, they first encountered a consent form, which described the purpose, description, and potential benefits of the survey. A key element stressed was that participation was anonymous and when entering contacts and places visited, that the participants should use pseudonyms or nicknames because we were not interested in the actual contacts or places 
visited, just that if they can remember them. Examples of pseudonyms were given as helpful suggestions (e.g., my best friend, my favorite bar).

The experiment began with definition of "contacts" and "places". Contacts were defined as "being in the same proximity within six feet with anybody for at least a duration of 10 minutes". An illustration of two people within a six feet radius was presented to give an example. A place was defined as a "populated location visited outside your home. Examples include 'grocery store, doctor's office, work building, restaurant, night club, etc.'" After students agreed upon the definitions of contact and places, they were randomized into one of the three independent conditions (see "Measures" for a description) for eliciting contacts and places. After reporting contacts and places, participants were all given items for control variables. These items included beliefs regarding COVID-19, mood, well-being, ability, employment.

\subsection{Measures}

\subsection{Contact Elicitation}

Contact elicitation was the key manipulation. There were three independent conditions. In the baseline approach, participants were asked to report their close social contacts motivated by wording from the General Social Survey [22]. The question was adapted to reflect places visited as well. The "places" question read:

Please take several minutes to think back from the last four days. Can you recall all of the places you have visited during those 4 days? Please use pseudonyms as we are not interested in the actual place.

The "contact" question similarly read:

Please take a few minutes to think back from the last four days. Can you recall all of the contacts you have made during those 4 days? If so, enter each name below. Please use pseudonyms as we are not interested in their real names.

For the retrieval cue approach, we followed procedures laid out by Hiesh [10]. Before eliciting contacts, participants were instructed to look at their (1) cellphone calls and texts from the last four days, (2) last 30 emails, and (3) recent posts, tags, and messages they have sent or received on their preferred social media (e.g., Facebook, Twitter, etc.) as retrieval cues. Then, the same two questions above were provided to elicit contacts and places.

Finally, for the context-based approach, four activity foci were activated to elicit contacts and places: (1) homelife, (2) worklife \& resources, (3) leisure, and (4) other. More specifically, the questions read:

1. Homelife: Many people have permanent residencies where they live with family members, partners, and/or friends.

a. Can you identify all of the homes you have visited within the past 4 days?

b. Can you identify all of the people that you have had any contact regarding homelife within the past 4 days? These include people who you live with and people who have visited your home. These also include people who's home you have visited.

2. Worklife \& resources: Many people must travel to places to gather resources for daily life. These include things like their workplace and shopping for food, clothes, gas, medical prescriptions, home supplies, or various technologies (e.g., a new phone).

a. Can you identify all of the places that you have visited regarding worklife/resources within the past 4 days?

b. Can you identify all of the people that you have had any contact regarding worklife/resources within the past 4 days? These include people who you work closely with and people you interact with to get the resources you need. 
3. Leisure: Many people engage in leisure activities with other people outside of work. These include things like going to churches, social clubs, restaurants, bars, gyms, going to the beach, and public events.

a. Can you identify all of the places that you have visited regarding leisure within the past 4 days?

b. Can you identify all of the people that you have had any contact regarding leisure within the past $\mathbf{4}$ days? These include people who you engage with in leisure activities outside of work.

4. Other: Are there any other places you have visited that you have not entered yet?

a. Can you identify all of the other places that you have visited within the past 4 days?

b. Can you identify more contacts within the past 4 days?

In all three conditions, participants provided contacts and places in a text box for convenience. Each participants' text was counted individually by the authors to check for duplicates (e.g., a participant lists the same contact or place twice) and general adherent to the survey procedures (e.g., removing participants that did not follow instructions). The final two dependent variables were the amount of unique contacts and places elicited by each participant.

\subsubsection{Control Variables}

Past research on individual level factors and network alter recall can help give guidance for additional control variables to include. Our review has identified such factors as gender [23], age [10], race [10], mood [24], well-being [13], and physical functioning [25]. Standard items were used to gather information on demographics like gender, age, and race. For mood (e.g., I am generally in a happy mood) we included nine items $(\alpha=0.91)$ from a scale developed by [26]. For well-being (e.g., How satisfied are you with your current well-being?), we used the nine items $(\alpha=0.86)$ provided by Shea et al. [13]. Physical functioning (e.g., Do you have difficulty walking?) was measuring using five items ( $\alpha$ $=0.81$ ) from Ross and Mirowsky [27].

Finally, we sought to include three single item questions regarding attitudes COVID-19 and masks. It may be the case that individuals who have more relaxed views on the risk of COVID-19 and the efficacy of masks may be more likely to engage in interpersonal contacts and visit more places. The questions included were "On a scale of 1-10, how serious of a public health threat is COVID-19?", "How worried are you about getting COVID-19?" and "Do you think wearing a mask helps to reduce the spread of COVID-19?" All items are listed in the Appendix A for reference.

\subsection{Analysis}

Because both dependent variables represent 'count' data (i.e., contacts and places visited) with skewed distributions, we opted for a negative binomial regression model. A negative binomial regression was chosen over a Poisson model particularly because the variance was greater than the mean for both contacts and places [28]. Robust standardized errors were used for more precise estimates of coefficient values. Effect size measures were calculated following Coxe's [29] method for calculating standardized mean differences (SMD): 0.20 - 0.50, small; $0.50-0.80=$ medium; and above $0.80=$ large. In step 1, only the key contact elicitation variable was entered. In step 2, the control variables were added to see if they impacted the association between method of contact elicitation and the two dependent variables. All analysis was performed using Stata 16 [30].

\section{Results}

On average, the baseline approach elicited 9.28 contacts $(S D=8.69)$, the retrieval cue elicited $10.61(S D=7.68)$, and the context-approach elicited $13.13(S D=7.60)$. The number of places visited was lower. On average, the baseline approach elicited 5.72 places $(S D=2.30)$, the retrieval cue elicited $5.67(S D=3.08)$, and the context-approach elicited $8.27(S D=4.01)$. 
Table 2. Robust Negative Binomial Regression

\begin{tabular}{|c|c|c|c|c|c|c|}
\hline \multicolumn{7}{|c|}{ Contacts as dependent variable } \\
\hline Variable & Coef. (SE) & $p$ & $S M D$ & Coef. (SE) & $p$ & $S M D$ \\
\hline H1: Retrieval Cue & $.13(.10)$ & .18 & .22 & $.13(.09)$ & .13 & .22 \\
\hline H2: Context-Based & $.34^{* *}(.09)$ & .00 & $.62^{*}$ & $.34^{* *}(.09)$ & .00 & $.62^{*}$ \\
\hline \multicolumn{7}{|l|}{ Control Variables } \\
\hline Gender & & & & $.16^{*}(.07)$ & .03 & \\
\hline Age & & & & $.01(.01)$ & .53 & \\
\hline Asian & & & & $-.33^{*}(.15)$ & .03 & \\
\hline African American & & & & $-.34^{* *}(.10)$ & .00 & \\
\hline Hispanic & & & & $.07(.15)$ & .63 & \\
\hline Hawaiian/Pacific Islander & & & & $-.65^{* * *}(.10)$ & .00 & \\
\hline Other Race & & & & $.24^{\dagger}(.15)$ & .10 & \\
\hline Mood & & & & $.12^{*}(.06)$ & .04 & \\
\hline Well-being & & & & $-.02(.06)$ & .64 & \\
\hline Physical functioning & & & & $-.13(.20)$ & .52 & \\
\hline Mask efficacy & & & & $-.04(.04)$ & .34 & \\
\hline COVID-worry & & & & $-.08^{*}(.04)$ & .03 & \\
\hline COVID-seriousness & & & & $-.01(.02)$ & .56 & \\
\hline Intercept & $2.23^{* *}(.08)$ & .00 & & $2.04^{* *}(.51)$ & .00 & \\
\hline \multicolumn{7}{|c|}{ Places as dependent variable } \\
\hline Variable & Coef. (SE) & $p$ & $S M D$ & Coef. (SE) & $p$ & $S M D$ \\
\hline H1: Retrieval Cue & $-.01(.05)$ & .86 & -.02 & $-.03(.05)$ & .47 & -.08 \\
\hline H2: Context-Based & $.36^{* *}(.05)$ & 00 & $.89^{*}$ & $.33^{* *}(.05)$ & .00 & $.87^{*}$ \\
\hline
\end{tabular}

Control variables

\begin{tabular}{|c|c|c|}
\hline Gender & & $.22^{* *}(.05)$ \\
\hline Age & & $-.01(.01)$ \\
\hline Asian & & $.17(.06)$ \\
\hline African American & & $-.19^{* *}(.06)$ \\
\hline Hispanic & & $.06(.15)$ \\
\hline Hawaiian/Pacific Islander & & $.06(.15)$ \\
\hline Other Race & & $-.08(.09)$ \\
\hline Mood & & $.05(.04)$ \\
\hline Well-being & & $-.01(.05)$ \\
\hline Physical functioning & & $-.30 *(.14)$ \\
\hline Mask efficacy & & $-.02(.03)$ \\
\hline COVID-worry & & $-.04^{+}(.02)$ \\
\hline COVID-seriousness & & $-.01(.01)$ \\
\hline Intercept & $1.74^{* *}(.03)$ & $1.80^{* *}(.35)$ \\
\hline
\end{tabular}

Note: * $p \leq .01,{ }^{*} p \leq .05,+\leq .10$. SMD effect sizes: $.20-.50=$ small; $.50-.80=$ medium; and above .80 = large. For Gender, 1 = Male, $2=$ Woman. For race, the reference group is White.

$\mathrm{H} 1$ predicted that the retrieval cue approach, which instructs contacts to view their previous text messages, emails, and social media posts, would stimulate higher recall of contacts and places 
compared to a baseline approach (see Table 2). H1 was not supported when predicting contacts (Est. $=0.13, S E=0.10, p=0.13, S M D=0.22)$ or places $(E s t .=-0.03, S E=0.05, p=0.47, S M D=-0.08)$. As such, the null hypothesis assuming no differences between the baseline approach and retrieval cue was not rejected.

$\mathrm{H} 2$ predicted that the context-based approach, which primes participants to think about contacts and places with respect to homelife, worklife/resources, leisure, and other, would elicit more contacts and places than a baseline approach. $\mathrm{H} 2$ was supported. Compared to the baseline approach, the context-based approach garnered medium effect sizes when predicting contacts (Est. $=0.34, S E=0.09$, $p<0.01, S M D=0.62)$ and large effect sizes when predicting places $(E s t .=0.33, S E=0.05, p<0.01, S M D=$ $0.87)$.

RQ1 asked about differences between the retrieval cue approach and the context-based approach. To keep the effects of the control variables, coefficient contrasts were estimated using Bonferroni corrected z-scores with post-estimation (see Figure 1). In terms of contacts, the coefficient contrast $(\varsigma)$ between the retrieval cue and context-approach was significant $(\varsigma=0.21, S E=0.08, z=$ $2.54, p=0.03$ ). With respect to places, the coefficient contrast was significant as well and larger $(\zeta=$ $0.38, S E=0.06, z=6.26, p<0.01)$.

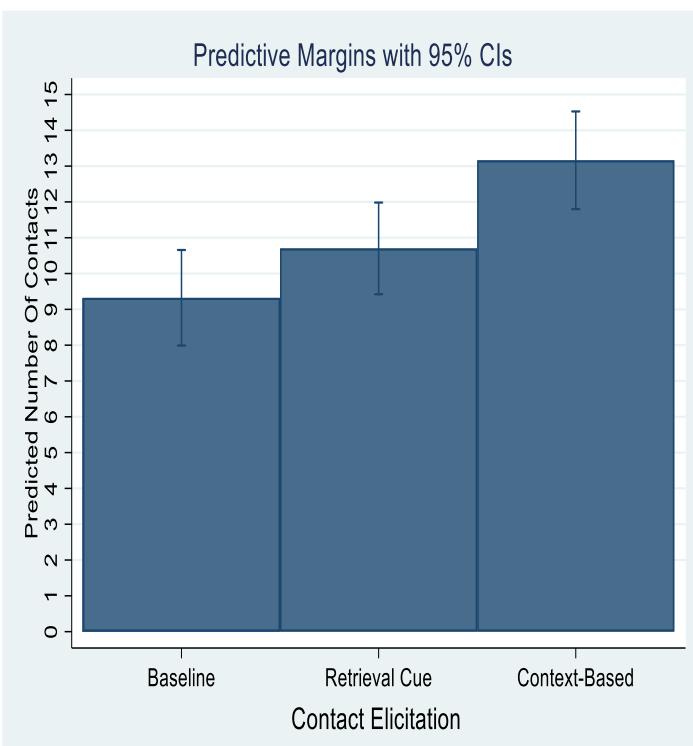

(a)

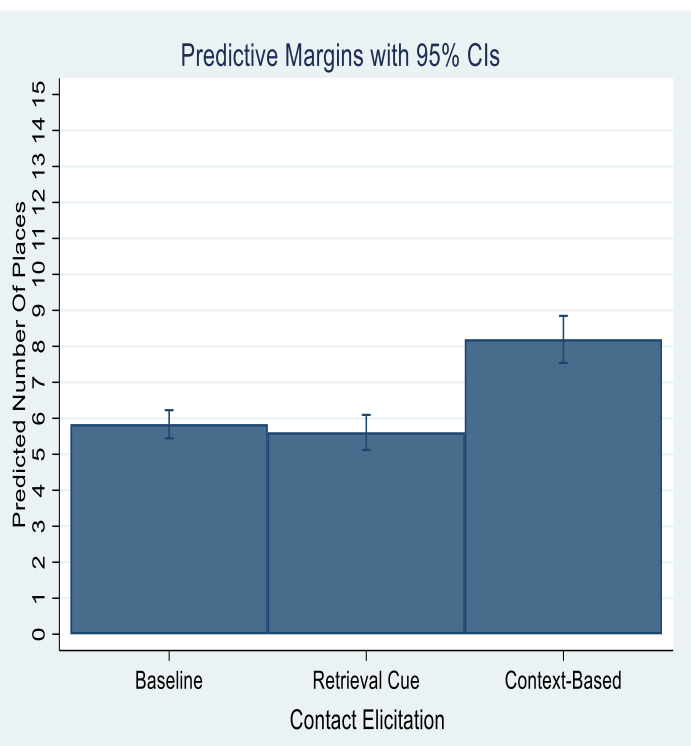

(b)

Figure 1. (a) Plots of post-estimation mean differences for contacts. (b) Plots of post-estimation mean differences for places.

\section{Discussion}

Valente [31] defines social network interventions as consisting of "behavior change programs that use social network data to identify specific people or groups to deliver and/or receive the behavior change program" (p. 146) and summarized six types of interventions. Contact tracing largely falls under the realm of network recruitment, where an ego's alters (i.e., contacts) are identified and recruited to quarantine and get tested for a possible infectious disease. Given the large costs associated with face-to-face interviews to conduct contact tracing, we sought to contribute to innovations in social network data collection methods for health-based interventions by developing a survey-based contact tracing instrument.

More specifically, the purpose of this research was to test the effectiveness of three different egocentric survey methods for eliciting contact tracing data: a (1) baseline, (2) retrieval cue, and (3) context-based approach. Overall, significantly more contacts and places were elicited when participants were randomized into the context-based condition, which primed users on activity foci of homelife, worklife/resources, leisure, and other. Theoretically, the results provide support field theory's suggestion that better recall can be stimulated when relevant activity foci are triggered. 
Practically, the study begins to shed light on more effective ways to design egocentric contact tracing surveys. Each of these realms are discussed further below.

There are few examples of research that test somewhat competing methods of eliciting interaction-based contacts. The current study nearly replicated Hsieh's [10] study which found that a retrieval cue approach elicits more contacts than a baseline approach $(p=0.13)$. However, a few caveats much be mentioned before any premature conclusions. First, Hsieh [10] elicited affective contacts (i.e., contacts whom the ego discusses important matters with) not interaction contacts as compared to the current study. Second, there could be selection bias in that the current study only looked at college students from a single university. Because different age groups and generations tend to use technology in different ways and have different attitudes on such technology (e.g., [31]), it may be the case that the retrieval cue approach may not be as effective with younger participants. Future research may be served well by including a more generalizable sample with larger diversity of age groups, locations, and socio-economic status.

Nevertheless, the results found that the context-based approach elicited more contacts and places visited than the baseline and retrieval cue approaches. Following field theory, Corman and Scott [32] theorize a similar network reticulation theory for explaining the relationship between perceived and observable networks (see also [33]). Again, the key here is to activate relevant activity foci to stimulate network recall of observable interactions. However, a key limitations for adapting the context-based approach of Bidart and Charbonneau [17] is determining relevant activity foci. Similar survey adaptations of the approach are typically contextual, including foci relevant to education paths, youth foster care, and individuals who live alone (see [17], fn. 7). The four foci here are by no means a final set and will need to be continually refined in order to create a gold-standard survey to elicit alters for contact tracing. Finally, just because an instrument elicits more contacts and places, does not necessarily means it performs the same as a face-to-face interview. Further studies might consider comparing survey-based approaches to more intensive qualitative approaches to really see how much they differ.

\section{Conclusions}

The main contribution the current research makes is with respect to developing innovations in social network data collection methods for one of the oldest type of relational health-based interventions: contact tracing. The current results provide support for the context-based approach, which first attempts to trigger relevant activity foci and then elicits contacts corresponding to such foci. Participants in the context-based were significantly more likely to elicit more contacts and places visited compared to traditional baseline approaches and the retrieval cue approach.

To put it simply, some communicative interactions may appear on the surface as random (e.g., a conversation with a bartender), which may make it difficult for individuals to remember them. However, this only appears to be the case if they are not viewed as situated action and stripped away from relevant activity foci (e.g., leisure $\rightarrow$ going to a bar). If it is true that contact tracing is only as good as the data that can be extracted, then making strides at developing a reliable and valid survey for easier and faster deployment can significantly impact public health with respect to infectious diseases that diffuse through interactive-based contact.

Supplementary Materials: Anonymous link to dataset:

https://osf.io/u7bda/?view_only=0394755b34a843808ac5cf34e8369b11

Author Contributions: Conceptualization, A.P.; methodology, AP. and C.H..; software, A.P.; validation, A.P.; formal analysis, A.P..; investigation, A.P. and C.H..; resources, A.P..; data curation, A.P. and C.H..; writing - original draft preparation, A.P. and C.H.; writing - review and editing, A.P. and C.H.; visualization, A.P.; supervision, A.P..; project administration, A.P..; funding acquisition, A.P. All authors have read and agreed to the published version of the manuscript.

Funding: This research was supported by the National Center for Research Resources and the National Center for Advancing Translational Sciences, National Institutes of Health, through Grant 
UL1TR001998. The content is solely the responsibility of the authors and does not necessarily represent the official views of the NIH.

Conflicts of Interest: The authors declare no conflict of interest.

\section{Appendix A}

Questionnaire

Race

Which option best describes your race?

a) American Indian or Alaska Native

b) Asian

c) Black or African American

d) Hispanic or Latino Origin

e) Native Hawaiian or Other Pacific Islander

f) White

g) Other

Gender

Which option best describes your gender?
a) man
b) woman
c) non-binary
d) prefer not to disclose
e) prefer to self-describe

Mood: (Rholes et al. [26]; Cronbach's alpha $=0.91$ )
a) I am generally in an elated mood.
b) I am generally in a cheerful mood.
c) I am generally in a happy mood.
d) I am generally in a good mood.
e) I am generally in a sad mood.
f) I am generally in a discouraged mood.
g) I am generally in a depressed mood.
h) I am generally in a bad mood.
The answer categories were
1) Strongly disagree
2) Disagree
3) Neither agree nor disagree
4) Agree
5) Strongly agree

Well-being: (Shea et al. [13]; Cronbach's alpha $=0.86$ )
a) How satisfied are you with your standard of living?
b) How satisfied are your with your health?
c) How satisfied are you with what you are achieving in life?
d) How satisfied are you with your personal relationships?
e) How satisfied are you with how safe you feel?
f) How satisfied are you with feeling part of your community?
g) How satisfied are you with your future security?
h) How satisfied are you with the amount of time you have to do the things that you like doing? The answer categories were
1) Strongly dissatisfied
2) Dissatisfied
3) Neither satisfied nor dissatisfied
4) Satisfied
5) Strongly satisfied 
Physical functioning: (Ross $\mathcal{E}$ Mirowsky [27]; Cronbach's alpha $=0.81$ )

Do you have any trouble or difficulties

a) walking?

b) using stairs or inclines?

c) standing or sitting for long periods?

d) using your fingers to grasp or handle?

e) lifting or carrying something as heavy as 10 pounds?

The answer categories were

1) $\mathrm{No}$

2) Yes, some

3) Yes, a great deal

Mask efficacy

Do you think wearing a mask helps to reduce the spread of COVID-19?

The answer categories were

1) No, it increases the spread

2) No, it does nothing

3) Not sure

4) Yes, some

5) Yes, a lot

COVID: Worry

How worried are you about getting COVID-19?

The answer categories were

1) Not worried at all

2) Somewhat worried

3) A little worried

4) Very worried

COVID: Seriousness

On a scale of 1-10, how serious of a public health threat is COVID-19?

The answer categories were $1=$ being no threat and $10=$ being very serious

\section{References}

1. Eames, K.T.D.; Keeling, M.J. Contact tracing and disease control. Proceedings of the Royal Society of London. Series B: Biological Sciences 2003, 270, 2565-2571, doi:doi:10.1098/rspb.2003.2554.

2. McCarty, C.; Lubbers, M.J.; Vacca, R.; Molina, J.L. Conducting personal network research: A practical guide; Guilford Publications: New York, 2019.

3. Perry, B.L.; Pescosolido, B.A.; Borgatti, S.P. Egocentric network analysis: Foundations, methods, and models; Cambridge University Press: Cambridge, MA, 2018; DOI: 10.1017/9781316443255.

4. Pilny, A.; Xiang, L. The impact of contact-tracing on COVID-19: An egocentric agent-based model. In Proceedings of Sunbelt International Network for Social Network Analysis, Paris, FR.

5. Jamerson, J. U.S. falling short on needed contact tracers, experts say. The Wall Street Journal 2020.

6. McLuhan, M. The medium is the message. MIT Press: Cambridge, MA, 1964.

7. Alalwan, A.A. Mobile food ordering apps: An empirical study of the factors affecting customer e-satisfaction and continued intention to reuse. International Journal of Information Management 2020, 50, 28-44, doi:https://doi.org/10.1016/j.ijinfomgt.2019.04.008.

8. Brewer, D.D. Forgetting in the recall-based elicitation of personal and social networks. Social Networks 2000, 22, 29-43, doi:https://doi.org/10.1016/S0378-8733(99)00017-9. 
9. Brashears, M.E.; Quintane, E. The microstructures of network recall: How social networks are encoded and represented in human memory. Social Networks 2015, 41, 113-126, doi:https://doi.org/10.1016/j.socnet.2014.11.003.

10. Hsieh, Y.P. Check the phone book: Testing information and communication technology (ICT) recall aids for personal network surveys. Social Networks 2015, 41, 101-112, doi:https://doi.org/10.1016/j.socnet.2014.11.006.

11. Omodei, E.; Brashears, M.E.; Arenas, A. A Mechanistic Model of Human Recall of Social Network Structure and Relationship Affect. Scientific Reports 2017, 7, 17133, doi:10.1038/s41598-017-17385-z.

12. Hlebec, V.; Ferligoj, A. Respondent mood and the instability of survey network measurements. Social Networks 2001, 23, 125-140, doi:https://doi.org/10.1016/S03788733(01)00037-5.

13. Shea, C.T.; Menon, T.; Smith, E.B.; Emich, K. The affective antecedents of cognitive social network activation. Social Networks 2015, 43, 91-99, doi:https://doi.org/10.1016/j.socnet.2015.01.003.

14. van der Poel, M.G.M. Delineating personal support networks. Social Networks 1993, 15, 49-70, doi:https://doi.org/10.1016/0378-8733(93)90021-C.

15. Mossong, J.; Hens, N.; Jit, M.; Beutels, P.; Auranen, K.; Mikolajczyk, R.; Massari, M.; Salmaso, S.; Tomba, G.S.; Wallinga, J., et al. Social Contacts and Mixing Patterns Relevant to the Spread of Infectious Diseases. PLOS Medicine 2008, 5, e74, doi:10.1371/journal.pmed.0050074.

16. Tulving, E. Cue-Dependent Forgetting: When we forget something we once knew, it does not necessarily mean that the memory trace has been lost; it may only be inaccessible. American Scientist 1974, 62, 74-82.

17. Bidart, C.; Charbonneau, J. How to Generate Personal Networks: Issues and Tools for a Sociological Perspective. Field Methods 2011, 23, 266-286, doi:10.1177/1525822X11408513.

18. Milardo, R.M. Theoretical and Methodological Issues in the Identification of the Social Networks of Spouses. Journal of Marriage and Family 1989, 51, 165-174, doi:10.2307/352377.

19. Feld, S.L. The Focused Organization of Social Ties. American Journal of Sociology 1981, 86, 10151035, doi:10.1086/227352.

20. Brashears, M.E. Humans use Compression Heuristics to Improve the Recall of Social Networks. Scientific Reports 2013, 3, 1513, doi:10.1038/srep01513.

21. Bahl, R.; Eikmeier, N.; Fraser, A.; Junge, M.; Keesing, F.; Nakahata, K.; Wang, L.Z. Modeling covid-19 spread in small colleges. arXiv preprint arXiv:2008.09597 2020.

22. Burt, R.S. Network items and the general social survey. Social Networks 1984, 6, 293-339, doi:https://doi.org/10.1016/0378-8733(84)90007-8.

23. Brashears, M.E.; Hoagland, E.; Quintane, E. Sex and network recall accuracy. Social Networks 2016, 44, 74-84, doi:https://doi.org/10.1016/j.socnet.2015.06.002.

24. Hlebec, V.; Ferligoj, A. Respondent mood and the instability of survey network measurements. Social Networks 2001, 23, 125-140, doi:https://doi.org/10.1016/S03788733(01)00037-5.

25. Bertera, E.M. Physical Activity and Social Network Contacts in Community Dwelling Older Adults. Activities, Adaptation \& Aging 2003, 27, 113-127, doi:10.1300/J016v27n03_08.

26. Rholes, W.S.; Riskind, J.H.; Lane, J.W. Emotional states and memory biases: Effects of cognitive priming and mood. Journal of Personality and Social Psychology 1987, 52, 91-99, 
doi:10.1037/0022-3514.52.1.91.

27. Ross, C.E.; Mirowsky, J. Does Employment Affect Health? Journal of Health and Social Behavior 1995, 36, 230-243, doi:10.2307/2137340.

28. Wilcox, R. Chapter 10 - Robust Regression. In Introduction to Robust Estimation and Hypothesis Testing (Third Edition), Wilcox, R., Ed. Academic Press: Boston, 2012; https://doi.org/10.1016/B978-0-12-386983-8.00010-Xpp. 471-532.

29. Coxe, S. Effect size measures for nonlinear count regression models. In Proceedings of Poster presented at the American Statistical Association Conference on Statistical Practice, Portland, Oregon.

30. StataCorp Stata Statistical Software: Release 16, StataCorp LLC: College Station, TX, 2019.

31. Valente, T.W. Social networks and health: Models, methods, and applications; Oxford University Press: Oxford, 2010.

32. Olson, K.E.; O’Brien, M.A.; Rogers, W.A.; Charness, N. Diffusion of Technology: Frequency of use for Younger and Older Adults. Ageing International 2011, 36, 123-145, doi:10.1007/s12126-010-9077-9.

33. Pilny, A.; Proulx, J.D.; Dinh, L.; Bryan, A.L. An Adapted Structurational Framework for the Emergence of Communication Networks. Communication Studies 2017, 68, 72-94, doi:10.1080/10510974.2016.1262886.

34. Corman, S.R.; Scott, C.R. Perceived Networks, Activity Foci, and Observable Communication in Social Collectives. Communication Theory 2006, 4, 171-190, doi:10.1111/j.14682885.1994.tb00089.x. 